\title{
Primary Immunodeficiency Disorders: Where Do We Stand?
}

\section{Anju Gupta ${ }^{1}$}

Received: 3 July 2019 / Accepted: 8 July 2019 / Published online: 24 July 2019

(C) Dr. K C Chaudhuri Foundation 2019

Primary immunodeficiency disorders (PID) are a group of nearly 350 disorders with varying manifestations. Early diagnosis and appropriate treatment are crucial for acceptable long-term outcomes. With minimum prevalence and incidence rates of $6 / 1,00,000$ population and 1/4,000 live births, we should expect at least 80,000 patients with clinically significant PID at any time and addition of 4,000 more to this pool every year [1]. Considering these as life-long diseases, the load is tremendous and the responsibility is significant for the physicians.

In this issue of the Journal, the study by Gupta et al. describes 120 patients diagnosed with PID using flow cytometry over duration of $3 \mathrm{y}$ from a premier health care setting in India [2]. The authors have presented a different disease profile compared to the previously published reports both from India and the West $[3,4]$. The predominance of combined immunodeficiency and phagocytic defects with relative paucity of primary antibody defects is likely to be related to inclusion of chronic granulomatous disease (CGD) carriers and non-inclusion of adults and non-infectious manifestations. Frequency of leucocyte adhesion defect (LAD) is significantly high in this series, either due to referral pattern or due to screening of newborns with delayed cord fall. It would be interesting to know what proportion of newborns with delayed cord separation turned out to have LAD. Absence of complement defects, autoinflammatory disorders and disorders of innate immunity are possibly related to exclusion of patients with clinical manifestations other than recurrent infections and lack of appropriate diagnostic modalities. Here it is pertinent to remember that manifestations of PID can be protean and include autoimmunity, autoinflammation, recurrent infections

Anju Gupta

anjupgi@gmail.com

1 Department of Pediatrics, Postgraduate Institute of Medical Education and Research, Chandigarh 160012, India and malignancy and therefore can present to different subspecialists including adult physicians.

Clinical suspicion and basic laboratory investigations can help us pinpoint a group of PIDs. However, as the spectrum of PIDs is wide, reliance on clinical and basic laboratory parameters for diagnosis is largely inadequate. In this context, the role of genetic analysis and flow cytometry is important. Although the former is foolproof, it is expensive, not easily available and associated with longer turnaround time. Flow cytometry acts as a bridge between basic laboratory tests and genetic diagnosis [5]. It helps in the diagnosis of several PIDs by evaluation of specific cell populations or subpopulations as in X-linked agammaglobulinemia (XLA) and severe combined immunodeficiency (SCID), various cellular proteins as in $\mathrm{LAD}$, biologic effects associated with immune defects as in Common Variable Immune Deficiency (CVID) and functional immune defects as in CGD. However we must also remember certain caveats of this method. Low perforin expression and CD107a upregulation in NK or cytotoxic T cells have a low positive predictive value for diagnosis of primary HLH [6]. Similarly, leucopenia and lymphopenia in an infant with severe sepsis does not always mean SCID. Percentage of naïve T cells may be useful in such a situation [5].

As a country, we have a long way to go in this field. Lack of awareness, sparse diagnostic modalities and lack of coordination between all the stakeholders are the major issues. This study is a welcome step in this direction. It helps in increasing awareness among physicians regarding the clinical spectrum and evaluation of PID. Establishing a nationwide registry and improving facilities for basic and genetic diagnosis should be the way forward. More importantly, our research should focus on formulating costeffective evaluation strategies for the 'at risk' children so that limited resources are used judiciously.

\section{Compliance with Ethical Standards}

Conflict of Interest None. 


\section{References}

1. Mahlaoui N, Gathmann B, Kindle G, Ehl S, on behalf of the ESID Registry Working Party Steering Committee and the ESID Society. The European Society for Immunodeficiencies (ESID) Registry: recent advancements in the epidemiology of primary immunodeficiencies and how does that translate in clinical care. Rare Dis Orphan Drugs. 2014;1:25-7.

2. Gupta D, Thakral D, Kumar P, et al. Primary immunodeficiency disorders among north Indian children. Indian J Pediatr. 2019. https://doi.org/10.1007/s12098-019-02971-y.

3. Modell V, Orange JS, Quinn J, Modell F. Global report on primary immunodeficiencies: 2018 update from the Jeffrey Modell centers network on disease classification, regional trends, treatment modalities, and physician reported outcomes. Immunol Res. 2018;66:367-80

4. Jindal AK, Pilania RK, Rawat A, Singh S. Primary immunodeficiency in India: a situational review. Front Immunol. 2017;8:714.

5. Madkaikar MR, Shabrish S, Kulkarni M, et al. Application of flow cytometry in primary immunodeficiencies: experience from India. Front Immunol. 2019;19:1248.

6. Rubin TS, Zhang K, Gifford C, et al. Perforin and CD107a testing is superior to NK cell function testing for screening patients for genetic HLH. Blood. 2017;129:2993-9.

Publisher's Note Springer Nature remains neutral with regard to jurisdictional claims in published maps and institutional affiliations. 